HETEROCYCLES, Vol. 91, No. 4, 2015, pp. 782 - 794. @ 2015 The Japan Institute of Heterocyclic Chemistry Received, 6th February, 2015, Accepted, 9th March, 2015, Published online, 18th March, 2015 DOI: $10.3987 / C O M-15-13188$

\title{
A SYNTHESIS OF LAMELLARINS VIA REGIOSELECTIVE ASSEMBLY OF 1,2,3-DIFFERENTIALLY SUBSTITUTED 5,6-DIHYDROPYRROLO[2,1-a]ISOQUINOLINE CORE
}

\section{Tsutomu Fukuda, Daichi Sato, and Masatomo Iwao*}

\author{
Division of Chemistry and Materials Science, Graduate School of Engineering, \\ Nagasaki University, 1-14 Bunkyo-machi, Nagasaki 852-8521, Japan; E-mail: \\ iwao@nagasaki-u.ac.jp
}

\begin{abstract}
A modular synthesis of the marine natural products lamellarins has been developed. The key reactions utilized are C3-selective Vilsmeier-Haack formylation followed by iterative bromination/cross-coupling of the 5,6-dihydropyrrolo[2,1-a]isoquinoline core. The 1,2-diaryl-5,6-dihydropyrrolo$[2,1-a]$ isoquinoline-3-carbaldehyde thus synthesized was readily converted to the lamellarin skeleton by mean of palladium-catalyzed oxidative lactonization.
\end{abstract}

\section{INTRODUCTION}

Lamellarins constitute an important class of natural products of marine origin. ${ }^{1}$ Since the first isolation of lamellarins A-D from Lamellaria sp. by Faulkner in $1985,{ }^{2 a}$ more than 50 lamellarins (A-Z, $\alpha-\chi, A 1-$ A6, and $\mathrm{O} 1-\mathrm{O} 2$, including their acetate and sulfate derivatives) have been isolated from marine organisms such as tunicates, sponges, and prosobranches. ${ }^{2}$ With a very few exceptions, lamellarins possess a unique 14-phenyl-6H-[1]benzopyrano[4',3':4,5]pyrrolo[2,1-a]isoquinolin-6-one ring system (Figure 1). Furthermore, these lamellarins exhibit various interesting biological activities including potent antiproliferative activity against several cancer cell lines, ${ }^{2 \mathrm{~g}, \mathrm{~h}, \mathrm{j}, \mathrm{k}, \mathrm{m}, \mathrm{n}, 3}$ multi-drug resistance (MDR) reversal activity, ${ }^{2 \mathrm{n}, 3 \mathrm{a}}$ anti-HIV activity, ${ }^{2 \mathrm{j}, 3 \mathrm{c}, 4}$ topoisomerase $\mathrm{I}$ inhibitory activity, ${ }^{5}$ inhibition of mitochondrial function, ${ }^{6}$ and protein kinases inhibitory activity. ${ }^{7}$ Because of their unique structure and significant biological activities, lamellarins have attracted considerable attention from organic and medicinal chemists. Consequently, various synthetic methods for the preparation of lamellarins have been exploited so far. ${ }^{8}$ The synthetic methods can be classified broadly into two categories: one utilizes formation of the pyrrole core as the key step and the other employs regioselective functionalization of the pre-existing pyrrole core. Compared to the former approaches, the latter syntheses are more effective because a wide range of natural and artificial lamellarins can be obtained easily by simple modification of 


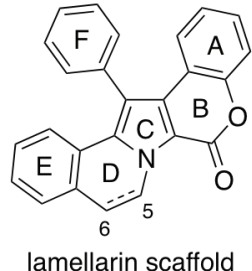

lamellarin scaffold

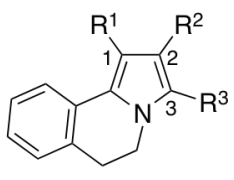

1,2,3-trisubstituted 5,6-dihydropyrrolo[2,1-a]isoquinoline

Figure 1

the aromatic building blocks substituted on the central pyrrole core (C-ring). Actually, the synthesis of lamellarins by using such modular approaches have been developed by several groups. ${ }^{8 \mathrm{~h}, \mathrm{j}, \mathrm{q}}$ Recently, we also developed a modular synthesis of lamellarins $\mathrm{L}$ and $\mathrm{N}$ via regioselective assembly of 3,4,5-differentially arylated pyrrole-2-carboxylates followed by construction of the D-ring of lamellarins by annulation between the pyrrole nitrogen and the lateral aromatic ring (E-ring). ${ }^{9}$ Turing our attention to the lamellarin scaffold again, we designed an alternative modular synthesis of lamellarins via a regioselective assembly of 1,2,3-trisubstituted 5,6-dihydropyrrolo[2,1-a]isoquinoline core (Figure 1). The present approach is similar to that of Álvarez in so far as the 5,6-dihydropyrrolo[2,1-a]isoquinoline is utilized as a platform of the lamellarin scaffold. However, the sequence of the ring construction and the functionalization of the key 5,6-dihydropyrrolo[2,1-a]isoquinoline core is different from those employed by Álvarez. ${ }^{8 j}$

\section{RESULTS AND DISCUSSION}

We selected lamellarins L (1) and N (2) as the targets of this synthetic approach. Our retrosynthetic analysis of 1 and 2 via 5,6-dihydropyrrolo[2,1-a]isoquinoline (5) is shown in Scheme 1. The conversion of lamellarin L triisopropyl ether (3) to the target lamellarins L (1) and N (2) have been established by

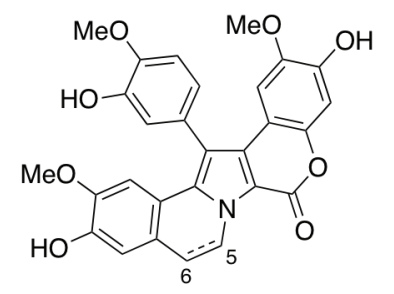

lamellarin $L(1)(5,6$-saturated) lamellarin $N(2)(5,6$-unsaturated)

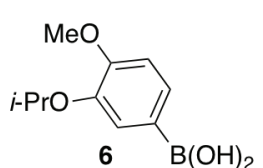
2. bromination
3. cross-coupling

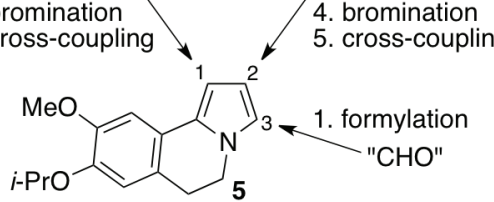

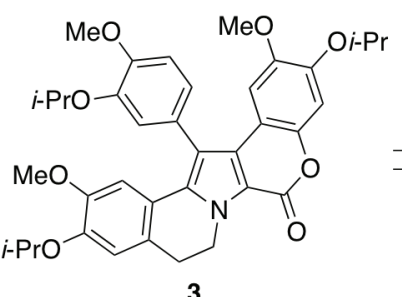

3

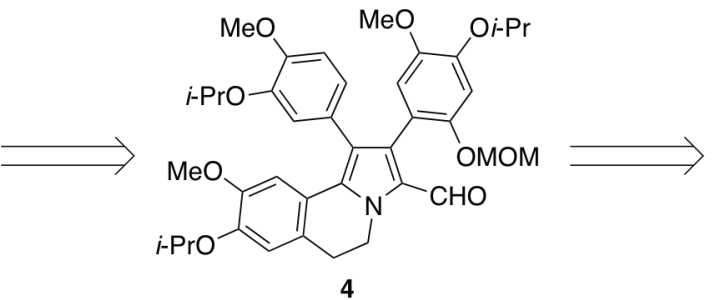<smiles>C=C</smiles>

Scheme 1 
us. ${ }^{8 k, 9}$ The compound (3) can be obtained by oxidation of 1,2-diaryl-5,6-dihydropyrrolo[2,1-a]isoquinoline-3-carbaldehyde (4) followed by acid-mediated methoxymethyl (MOM) deprotectionlactonization of the resulting phenolic acid. The intermediate (4) may be assembled from 5,6-dihydropyrrolo[2,1-a]isoquinoline (5) via following sequences: 1) C3-selective formylation of 5 under Vilsmeier-Haack reaction conditions, 2) C1-selective bromination followed by cross-coupling with arylboronic acid (6), 3) C2-selective bromination followed by cross-coupling with arylboronic acid (7). The key tricyclic compound (5) will be prepared in two steps from the phenethylamine (9) via the Paal-Knorr pyrrole synthesis and subsequent palladium-catalyzed intramolecular direct arylation of the pyrrole (8). ${ }^{8 p}$

Based on the retrosynthetic analysis, we performed the synthesis of 5,6-dihydropyrrolo[2,1-a]isoquinoline (5) at first. The starting material (9) was readily obtained by simple bromination of the known phenylethylamine $(\mathbf{1 0})^{8 \mathrm{k}}$ in good yield (Scheme 2). The compound (9) was isolated as its hydrobromide salt. ${ }^{8 d}$

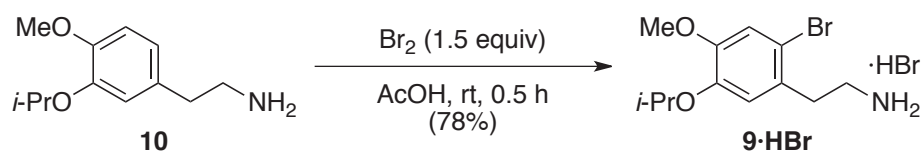

Scheme 2

We next examined the Paal-Knorr reaction of $\mathbf{9} \cdot \mathbf{H B r}$. The results are summarized in Table 1. Since D'Silva and Walker reported that treatment of the benzylamine hydrochlorides with 2,5-dimethoxytetrahydrofuran (DMT) in a mixture of pyridine, acetic acid, and water gave the corresponding pyrroles in moderate to good yields, ${ }^{10}$ we carried out the reactions under the similar

Table 1. Paal-Knorr reaction of the phenethylamine $(\mathbf{9} \cdot \mathbf{H B r})$ with 2,5-dimethoxytetrahydrofuran (DMT)

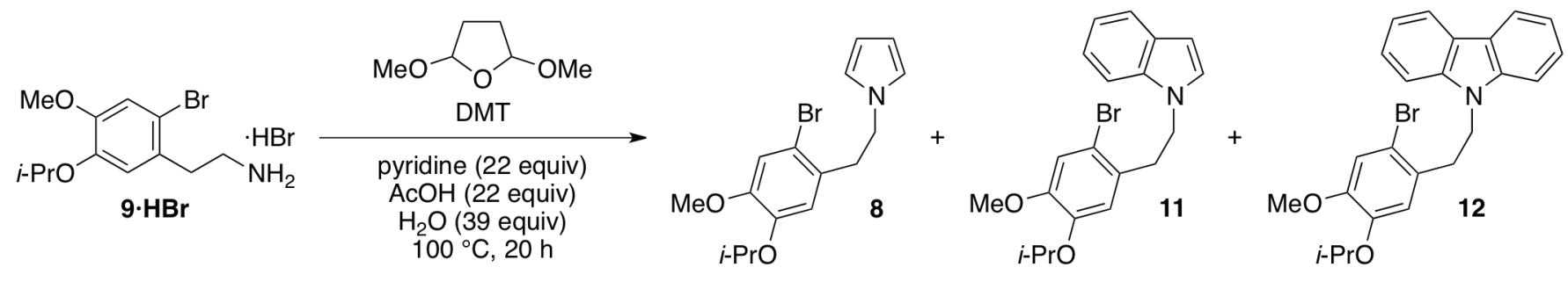

\begin{tabular}{ccccc}
\hline entry & DMT (equiv) & $\mathbf{8}(\%)^{\mathrm{a}}$ & $\mathbf{1 1}(\%)^{\mathrm{b}}$ & $\mathbf{1 2}(\%)^{\mathrm{b}}$ \\
\hline 1 & 1.0 & 93 & 0 & 0 \\
2 & 2.0 & 0 & 34 & 6 \\
3 & 3.0 & 0 & 15 & 19 \\
4 & 4.0 & 0 & 6 & 24 \\
\hline
\end{tabular}


conditions. The phenethylamine $(\mathbf{9} \cdot \mathbf{H B r})$ was treated with 1.0 equiv of DMT in a mixture of pyridine, acetic acid, and water at $100{ }^{\circ} \mathrm{C}$ for $20 \mathrm{~h}$, the desired pyrrole (8) was obtained in $93 \%$ yield (entry 1). When the amount of DMT was increased to 2.0 equiv, the indole (11) and the carbazole (12) were obtained in $34 \%$ and $6 \%$ yields, respectively (entry 2). These compounds may be formed by further condensation of 8 with DMT. ${ }^{11}$ The increasing amount of DMT under the similar conditions did not improve the yields of $\mathbf{1 1}$ or $\mathbf{1 2}$ due to formation of unidentified polymeric materials (entries 3 and 4).

Next, the palladium-catalyzed intramolecular direct arylation of the phenethylpyrrole (8) thus synthesized was tested (Scheme 3). Of our delight, the desired 5,6-dihydropyrrolo[2,1-a]isoquinoline (5) was obtained in good yield under the conditions previously established in our laboratories for a transformation of 3-[2-(2-bromophenyl)ethyl][1]benzopyrano[3,4-b]pyrrol-4(3H)-one to the pentacyclic lamellarin scaffold [10 mol\% of $\mathrm{Pd}\left(\mathrm{PPh}_{3}\right)_{4}, \mathrm{~K}_{2} \mathrm{CO}_{3}, N, N$-dimethylacetamide (DMA), $\left.125^{\circ} \mathrm{C}\right] .{ }^{8 \mathrm{p}}$

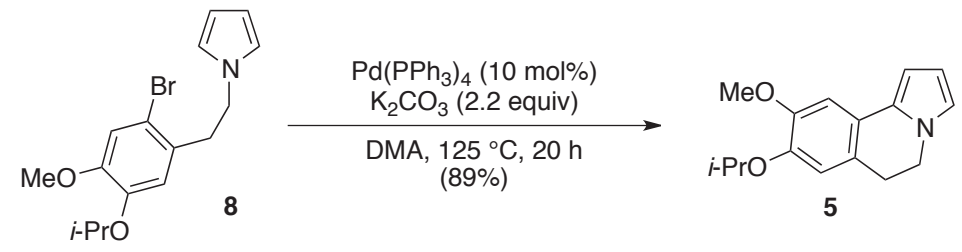

Scheme 3

With sufficient amount of the key 5,6-dihydropyrrolo[2,1-a]isoquinoline (5) in hand, we next focused on its conversion to lamellarins L (1) and N (2) (Scheme 4). Vilsmeier-Haack reaction of 5 using phosphoryl chloride in dimethylformamide (DMF) gave C3-selectively formylated $\mathbf{1 3}$ in $\mathbf{7 9 \%}$ yield. ${ }^{12}$ Subsequent reaction of $\mathbf{1 3}$ with $\mathrm{N}$-bromosuccinimide (NBS) in DMF gave compound 14 in $96 \%$ yield. The regioselectivity of this reaction was controlled by the electron-withdrawing formyl group. Similar regioselectivity has been reported for the bromination of 5,6-dihydropyrrolo[2,1-a]isoquinoline3-carboxylate by Álvarez. ${ }^{8 j} \quad$ Suzuki-Miyaura cross-coupling of $\mathbf{1 4}$ with arylboronic acid (6) proceeded smoothly to give the arylated compound (15) in good yield. Bromination of $\mathbf{1 5}$ with NBS followed by the cross-coupling with arylboronic acid (7) yielded 1,2-differentially arylated 5,6-dihydropyrrolo[2,1-a]isoquinoline-3-carbaldehyde (4) in good yield. For the conversion of 4 to $\mathbf{3}$, Pinnick oxidation $\left(\mathrm{NaClO}_{2}, 2\right.$-methyl-2-butene) of the aldehyde was tested at first. ${ }^{13}$ However, the reaction was sluggish and the starting material was recovered. The reason for the failure may be accounted for by the influence of the steric hindrance and the electron-donating nature of the pyrrole moiety adjacent to the formyl group. To avoid this problem, the reaction sequence was changed to perform MOM-deprotection at first, followed by oxidation. Thus, the aldehyde (4) was treated with concd $\mathrm{HCl}$ in $\mathrm{MeOH}$ to give 17. The successful conversion of this type of phenolic aldehyde to the lactone has been reported by Ruchirawat in their lamellarin synthesis. ${ }^{8 e}$ In fact, application of their 


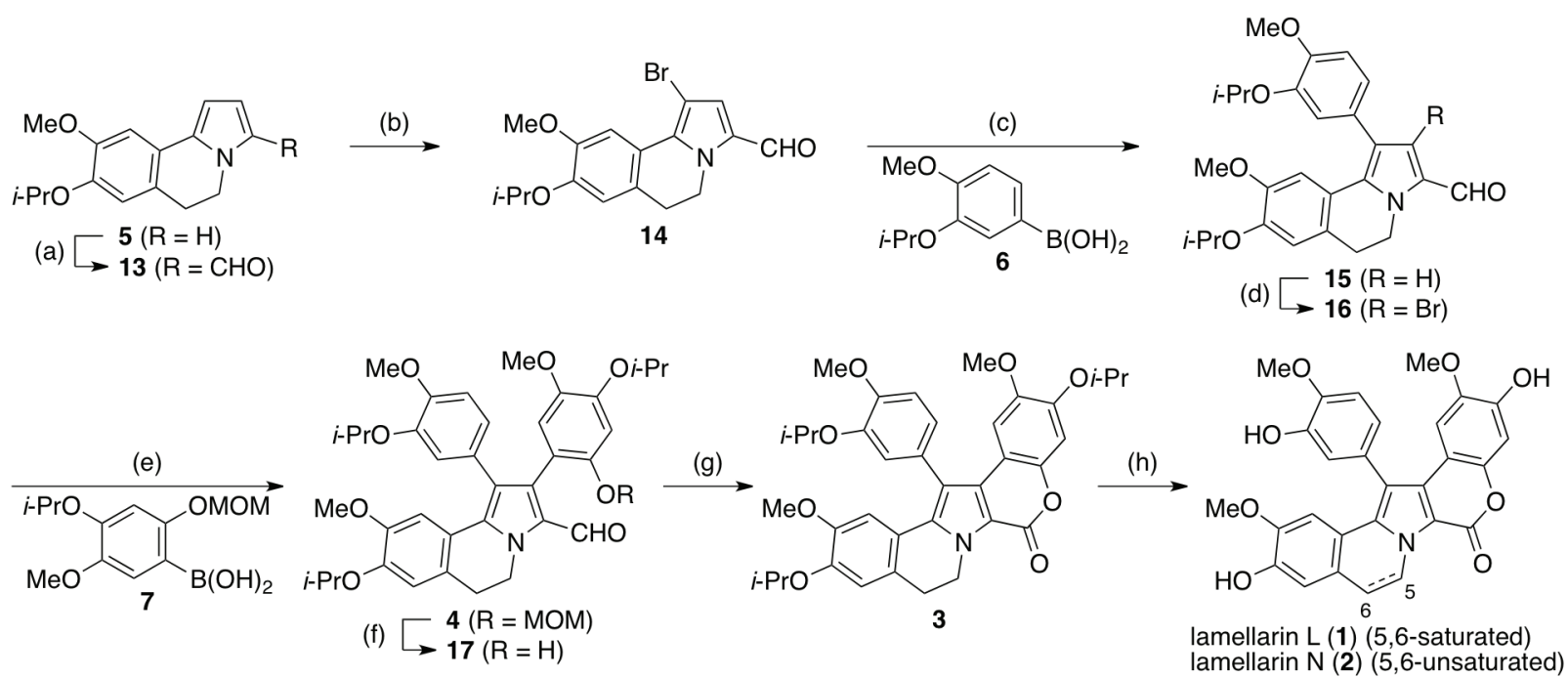

Scheme 4. Reagents and conditions: (a) $\mathrm{POCl}_{3}$ (1.1 equiv), DMF, $0{ }^{\circ} \mathrm{C}$ then $60{ }^{\circ} \mathrm{C}, 20 \mathrm{~h}(79 \%$ ); (b) NBS (1.0 equiv), THF, reflux, $3 \mathrm{~h}(96 \%)$; (c) 6 (1.5 equiv), $\mathrm{Pd}\left(\mathrm{PPh}_{3}\right)_{4}(10 \mathrm{~mol} \%), \mathrm{Na}_{2} \mathrm{CO}_{3}(6.6$ equiv), water, DME, reflux, $16 \mathrm{~h}$ (95\%); (d) NBS (1.5 equiv), DMF, $0{ }^{\circ} \mathrm{C}$ then $\mathrm{rt}, 2 \mathrm{~h}(76 \%)$; (e) 7 (1.5 equiv), $\mathrm{Pd}\left(\mathrm{PPh}_{3}\right)_{4}(10 \mathrm{~mol} \%), \mathrm{Na}_{2} \mathrm{CO}_{3}$ (6.6 equiv), water, DME, reflux, $19 \mathrm{~h}(82 \%)$; (f) concd $\mathrm{HCl}, \mathrm{MeOH}$, reflux, $2 \mathrm{~h}(73 \%)$; (g) $\mathrm{PhBr}$ (1.2 equiv), $\mathrm{Pd}(\mathrm{OAc})_{2}(10 \mathrm{~mol} \%), \mathrm{PPh}_{3}$ (30 mol\%), $\mathrm{K}_{2} \mathrm{CO}_{3}$ (1.1 equiv), DMF, $120^{\circ} \mathrm{C}, 13 \mathrm{~h}(52 \%)$; (h) see references 8k and 9.

conditions $\left[\mathrm{PhBr}\right.$ (1.2 equiv), $\mathrm{Pd}(\mathrm{OAc})_{2}(10 \mathrm{~mol} \%), \mathrm{PPh}_{3}(30 \mathrm{~mol} \%), \mathrm{K}_{2} \mathrm{CO}_{3}\left(1.1 \text { equiv), } \mathrm{DMF}, 120{ }^{\circ} \mathrm{C}\right]^{14}$ to $\mathbf{1 7}$ provided the desired lamellarin (3) in moderate yield. Since the conversion of $\mathbf{3}$ to lamellarins L (1) and $\mathrm{N}$ (2) has been established in our laboratories, ${ }^{8 \mathrm{k}, 9}$ the formal syntheses of these lamellarins were thus achieved.

In conclusion, we have developed a new modular synthesis of lamellarins via regioselective assembly of 1,2,3-differentially substituted 5,6-dihydropyrrolo[2,1-a]isoquinoline (5) followed by oxidative lactonization. This route may be applicable to the synthesis of a wide range of lamellarin derivatives by simple structural modification of 5,6-dihydropyrrolo[2,1-a]isoquinoline core and arylboronic acids.

\section{EXPERIMENTAL}

Melting points were determined with a Yanagimoto micro melting points apparatus and are uncorrected. IR spectra were obtained with a Thermo Nicolet Nexus 670 NT FT-IR instrument and are reported in terms of frequency of absorption $\left(\mathrm{cm}^{-1}\right)$. NMR spectra were recorded on a JEOL JNM-AL400 instrument (400 MHz for ${ }^{1} \mathrm{H}$ and $100 \mathrm{MHz}$ for ${ }^{13} \mathrm{C}$ ) or a Varian NMR System 500PS SN instrument (500 $\mathrm{MHz}$ for ${ }^{1} \mathrm{H}$ and $125 \mathrm{MHz}$ for ${ }^{13} \mathrm{C}$ ). Chemical shifts for ${ }^{1} \mathrm{H}$ NMR are expressed in parts per million (ppm) relative to tetramethylsilane $(\delta 0.0 \mathrm{ppm})$. Data for ${ }^{1} \mathrm{H}$ NMR spectra are reported as follows: chemical shift $(\delta$ ppm), multiplicity $(\mathrm{s}=$ singlet, $\mathrm{d}=$ doublet, $\mathrm{dd}=$ double of doublets, $\mathrm{t}=$ triplet, sep $=$ septet, $\mathrm{m}=$ multiplet, br $\mathrm{s}=$ broad signal), coupling constant $(\mathrm{Hz})$, and integration. Chemical shifts for ${ }^{13} \mathrm{C} \mathrm{NMR}$ are expressed in ppm relative to the following internal standards: $\mathrm{CDCl}_{3}$ (tetramethylsilane, $\delta$ 
$0.0 \mathrm{ppm}), \mathrm{DMSO}-d_{6}$ (DMSO- $\left.d_{6}, \delta 39.52 \mathrm{ppm}\right)$. Data for ${ }^{13} \mathrm{C}$ NMR spectra are reported in terms of chemical shift. High resolution mass spectra were recorded on a JEOL JMS-T100TD (direct analysis in real time mass spectrometry, DARTMS). Elemental analysis was performed for $\mathrm{C}, \mathrm{H}$, and $\mathrm{N}$ using a Perkin Elmer 2400II instrument. Column chromatography was conducted on silica gel 60N, 63-210 $\mu \mathrm{m}$ (Kanto Chemical Co., Inc.). Flash chromatography was conducted on silica gel 60N, 40-50 $\mu$ m (Kanto Chemical Co., Inc.) or Chromatorex NH-DM2035 silica gel (Fuji Silysia Chemical Ltd.).

2-(2-Bromo-5-isopropoxy-4-methoxyphenyl)ethylamine hydrobromide (9-HBr). A solution of bromine $(8.40 \mathrm{~g}, 52.6 \mathrm{mmol})$ in $\mathrm{AcOH}(30 \mathrm{~mL})$ was added dropwise to a solution of $\mathbf{1 0}^{8 \mathrm{k}}(7.33 \mathrm{~g}, 35.0$ $\mathrm{mmol})$ in $\mathrm{AcOH}(160 \mathrm{~mL})$ at $\mathrm{rt}$. After stirring for $0.5 \mathrm{~h}$ at $\mathrm{rt}$, the reaction mixture was concentrated under reduced pressure. The residue was diluted with EtOAc and the resulting white precipitate was corrected by filtration, washed with EtOAc, and dried under reduced pressure to give $\mathbf{9} \cdot \mathbf{H B r}$ as a colorless powder $(10.1 \mathrm{~g}, 78 \%)$. Mp 179.5-181.5 ${ }^{\circ} \mathrm{C}$ (sealed capillary). [lit. $\left.{ }^{8 \mathrm{~d}} \mathrm{Mp} 224{ }^{\circ} \mathrm{C}\right] . \quad \mathrm{IR}(\mathrm{KBr}): 2970$, $1594,1511,1266,1214,1171,1109,1031 \mathrm{~cm}^{-1}$. ${ }^{1} \mathrm{H}$ NMR $\left(400 \mathrm{MHz}, \mathrm{DMSO}-d_{6}\right): \delta 1.25(\mathrm{~d}, J=6.0 \mathrm{~Hz}$, 6H), 2.90-3.06 (m, 4H), $3.76(\mathrm{~s}, 3 \mathrm{H}), 4.56(\mathrm{sep}, J=6.0 \mathrm{~Hz}, 1 \mathrm{H}), 7.02(\mathrm{~s}, 1 \mathrm{H}), 7.15(\mathrm{~s}, 1 \mathrm{H}), 7.95$ (br s, $3 \mathrm{H}) .{ }^{13} \mathrm{C}$ NMR $\left(100 \mathrm{MHz}, \mathrm{DMSO}-d_{6}\right): \delta 21.8,32.7,38.6,55.9,70.7,113.7,116.1,117.6,128.1,146.3$, 149.7. HRMS m/z. Calcd for $\mathrm{C}_{12} \mathrm{H}_{19} \mathrm{BrNO}_{2}\left[(\mathrm{M}-\mathrm{Br})^{+}\right]$: 288.0599. Found: 288.0602. These spectroscopic data are in good agreement with those previously reported. ${ }^{8 \mathrm{~d}}$

Typical procedure for Paal-Knorr reaction of the phenethylamine (9·HBr) with DMT. Under an argon atmosphere, a mixture of $\mathbf{9} \cdot \mathbf{H B r}(150 \mathrm{mg}, 0.406 \mathrm{mmol})$, an appropriate amount of DMT, pyridine (723 $\mu \mathrm{L}, 8.94 \mathrm{mmol}), \mathrm{AcOH}(511 \mu \mathrm{L}, 8.94 \mathrm{mmol})$, and water $(286 \mu \mathrm{L}, 15.8 \mathrm{mmol})$ was heated in a sealed tube at $100{ }^{\circ} \mathrm{C}$ for $20 \mathrm{~h}$. After cooling to $\mathrm{rt}$, the mixture was diluted with EtOAc and the product was washed with $1 \mathrm{M}$ aqueous $\mathrm{HCl}$ and brine, dried over $\mathrm{Na}_{2} \mathrm{SO}_{4}$, and evaporated. The residue was purified by flash chromatography over Chromatorex NH-DM2035 silica gel (hexane-EtOAc $=30: 1$ ) to give 8 or a mixture of $\mathbf{1 1}$ and $\mathbf{1 2}$. Since all attempts to separation of $\mathbf{1 1}$ and $\mathbf{1 2}$ by flash chromatography were failed, the yields of $\mathbf{1 1}$ and $\mathbf{1 2}$ were estimated by integration of ${ }^{1} \mathrm{H}$ NMR absorption of H4 of $\mathbf{1 1}$ and H4 and H5 of $12\left(\delta \mathrm{H} 4\right.$ of 11: 7.59; $\delta \mathrm{H} 4$ and $\mathrm{H} 5$ of 12: 8.06). ${ }^{15} \quad$ The results were shown in Table 1.

1-[2-(2-Bromo-5-isopropoxy-4-methoxyphenyl)ethyl]-1H-pyrrole (8). Colorless granules. Mp 57.5-58.5 ${ }^{\circ} \mathrm{C}\left(\mathrm{Et}_{2} \mathrm{O}-\right.$ hexane). IR (KBr): 1507, 1253, 1211, 1034, 848, 729, $705 \mathrm{~cm}^{-1}$. ${ }^{1} \mathrm{H}$ NMR (400 $\left.\mathrm{MHz}, \mathrm{CDCl}_{3}\right): \delta 1.28(\mathrm{~d}, J=6.1 \mathrm{~Hz}, 6 \mathrm{H}), 3.06(\mathrm{t}, J=7.1 \mathrm{~Hz}, 2 \mathrm{H}), 3.82(\mathrm{~s}, 3 \mathrm{H}), 4.07(\mathrm{t}, J=7.1 \mathrm{~Hz}, 2 \mathrm{H})$, $4.33(\operatorname{sep}, J=6.1 \mathrm{~Hz}, 1 \mathrm{H}), 6.09(\mathrm{t}, J=2.1 \mathrm{~Hz}, 2 \mathrm{H}), 6.42(\mathrm{~s}, 1 \mathrm{H}), 6.57(\mathrm{t}, J=2.1 \mathrm{~Hz}, 2 \mathrm{H}), 7.02(\mathrm{~s}, 1 \mathrm{H})$. ${ }^{13} \mathrm{C}$ NMR $\left(100 \mathrm{MHz}, \mathrm{CDCl}_{3}\right): \delta 22.0,38.3,49.4,56.2,71.8,108.1,114.3,116.0,118.0,118.0,120.5$, 129.4, 146.6, 149.9. Anal. Calcd for $\mathrm{C}_{16} \mathrm{H}_{20} \mathrm{BrNO}_{2}$ : C, 56.82; H, 5.96; N, 4.14. Found: C, 57.03; H, $5.78 ; \mathrm{N}, 4.09$. 
1-[2-(2-Bromo-5-isopropoxy-4-methoxyphenyl)ethyl]-1H-indole (11). ${ }^{1} \mathrm{H} \mathrm{NMR}\left(400 \mathrm{MHz}, \mathrm{CDCl}_{3}\right): \delta$ $1.15(\mathrm{~d}, J=6.1 \mathrm{~Hz}, 6 \mathrm{H}), 3.13(\mathrm{t}, J=7.0 \mathrm{~Hz}, 2 \mathrm{H}), 3.81(\mathrm{~s}, 3 \mathrm{H}), 4.09(\mathrm{sep}, J=6.1 \mathrm{~Hz}, 1 \mathrm{H}), 4.35(\mathrm{t}, J=7.0$ $\mathrm{Hz}, 2 \mathrm{H}), 6.27(\mathrm{~s}, 1 \mathrm{H}), 6.41(\mathrm{~d}, J=3.1 \mathrm{~Hz}, 1 \mathrm{H}), 6.92(\mathrm{~d}, J=3.1 \mathrm{~Hz}, 1 \mathrm{H}), 7.02(\mathrm{~s}, 1 \mathrm{H}), 7.05-7.37(\mathrm{~m}, 3 \mathrm{H})$, $7.59(\mathrm{~d}, J=7.9 \mathrm{~Hz}, 1 \mathrm{H})$. HRMS $(m / z)$ Calcd for $\mathrm{C}_{20} \mathrm{H}_{23} \mathrm{BrNO}_{2}\left[(\mathrm{M}+\mathrm{H})^{+}\right]: 388.0912$. Found: 388.0919 . 9-[2-(2-Bromo-5-isopropoxy-4-methoxyphenyl)ethyl]-9H-carbazole (12). ${ }^{1} \mathrm{H}$ NMR (400 $\mathrm{MHz}$, $\left.\mathrm{CDCl}_{3}\right): \delta 1.05(\mathrm{~d}, J=6.1 \mathrm{~Hz}, 6 \mathrm{H}), 3.17(\mathrm{t}, J=7.1 \mathrm{~Hz}, 2 \mathrm{H}), 3.79(\mathrm{~s}, 3 \mathrm{H}), 3.98(\mathrm{sep}, J=6.1 \mathrm{~Hz}, 1 \mathrm{H}), 4.54$ $(\mathrm{t}, J=7.1 \mathrm{~Hz}, 2 \mathrm{H}), 6.28(\mathrm{~s}, 1 \mathrm{H}), 7.02(\mathrm{~s}, 1 \mathrm{H}), 7.16-7.44(\mathrm{~m}, 6 \mathrm{H}), 8.06(\mathrm{~d}, J=7.8 \mathrm{~Hz}, 2 \mathrm{H}) . \quad \mathrm{HRMS}(\mathrm{m} / \mathrm{z})$ Calcd for $\mathrm{C}_{20} \mathrm{H}_{23} \mathrm{BrNO}_{2}\left[(\mathrm{M}+\mathrm{H})^{+}\right]$: 438.1069. Found: 438.1097.

5,6-Dihydro-8-isopropoxy-9-methoxypyrrolo[2,1-a]isoquinoline (5). Under an argon atmosphere, a mixture of $8(2.71 \mathrm{~g}, 8.01 \mathrm{mmol}), \mathrm{K}_{2} \mathrm{CO}_{3}(2.43 \mathrm{~g}, 17.6 \mathrm{mmol})$ and $\mathrm{Pd}\left(\mathrm{PPh}_{3}\right)_{4}(925 \mathrm{mg}, 0.800 \mathrm{mmol})$ in DMA $(110 \mathrm{~mL})$ was heated at $125^{\circ} \mathrm{C}$ for $20 \mathrm{~h}$. After cooling to $\mathrm{rt}$, the mixture was diluted with water and extracted with $\mathrm{CH}_{2} \mathrm{Cl}_{2}$. The extract was washed with water and brine, dried over $\mathrm{Na}_{2} \mathrm{SO}_{4}$, and evaporated. The residue was purified by column chromatography over silica gel $60 \mathrm{~N}$ (hexane-EtOAc $=$ 10:1) to give 5 as a pale blue solid $(1.84 \mathrm{~g}, 89 \%)$. Recrystallization from $\mathrm{Et}_{2} \mathrm{O}$-hexane gave colorless granules. $\quad \mathrm{Mp} 70.5-71.5^{\circ} \mathrm{C}$. IR (KBr): $1505,1451,1265,1210,1112,864,714 \mathrm{~cm}^{-1}$. ${ }^{1} \mathrm{H}$ NMR (400 $\left.\mathrm{MHz}, \mathrm{CDCl}_{3}\right): \delta 1.37(\mathrm{~d}, J=6.1 \mathrm{~Hz}, 6 \mathrm{H}), 2.96(\mathrm{t}, J=6.6 \mathrm{~Hz}, 2 \mathrm{H}), 3.88(\mathrm{~s}, 3 \mathrm{H}), 4.04(\mathrm{t}, J=6.6 \mathrm{~Hz}, 2 \mathrm{H})$, $4.49(\mathrm{sep}, J=6.1 \mathrm{~Hz}, 1 \mathrm{H}), 6.19(\mathrm{t}, J=3.0 \mathrm{~Hz}, 1 \mathrm{H}), 6.39(\mathrm{~d}, J=3.5 \mathrm{~Hz}, 1 \mathrm{H}), 6.64(\mathrm{~s}, 1 \mathrm{H}), 6.72(\mathrm{~s}, 1 \mathrm{H})$,

$7.02(\mathrm{~s}, 1 \mathrm{H}) .{ }^{13} \mathrm{C}$ NMR $\left(100 \mathrm{MHz}, \mathrm{CDCl}_{3}\right): \delta 22.2,29.0,44.3,56.1,71.8,102.3,106.6,108.3,116.4$, 120.4, 122.8, 123.2, 130.0, 145.4, 149.8. Anal. Calcd for $\mathrm{C}_{16} \mathrm{H}_{19} \mathrm{NO}_{2}: \mathrm{C}, 74.68 ; \mathrm{H}, 7.44 ; \mathrm{N}, 5.44$. Found: C, 74.66; H, 7.74; N, 5.33.

5,6-Dihydro-8-isopropoxy-9-methoxypyrrolo[2,1-a]isoquinoline-3-carbaldehyde (13). Phosphorus oxychloride $(40.0 \mu \mathrm{L}, 0.429 \mathrm{mmol})$ was added dropwise to DMF $(3.2 \mathrm{~mL})$ at $0{ }^{\circ} \mathrm{C}$. After stirring for $1 \mathrm{~h}$ at $0{ }^{\circ} \mathrm{C}, 5$ (100 mg, $0.389 \mathrm{mmol}$ ) was added and then the mixture was allowed to warm to rt. The reaction mixture was heated at $60{ }^{\circ} \mathrm{C}$ and stirring was continued for $20 \mathrm{~h}$. After cooling to rt, the mixture was quenched with saturated aqueous $\mathrm{NaHCO}_{3}$ and the product was extracted with $\mathrm{CH}_{2} \mathrm{Cl}_{2}$. The extract was washed with water and brine, dried over $\mathrm{Na}_{2} \mathrm{SO}_{4}$, and evaporated. The residue was purified by column chromatography over silica gel $60 \mathrm{~N}$ (hexane-EtOAc $=3: 1$ ) to give $\mathbf{1 3}$ as a colorless solid (88.0 mg, 79\%). Recrystallization from $\mathrm{Et}_{2} \mathrm{O}$-hexane gave colorless needles. Mp 69-70 ${ }^{\circ} \mathrm{C}$. IR (KBr): $1641,1494,1402,1236,1156,1040,803 \mathrm{~cm}^{-1} \cdot{ }^{1} \mathrm{H}$ NMR $\left(400 \mathrm{MHz}, \mathrm{CDCl}_{3}\right): \delta 1.40(\mathrm{~d}, J=6.1$ $\mathrm{Hz}, 6 \mathrm{H}), 3.00(\mathrm{t}, J=6.9 \mathrm{~Hz}, 2 \mathrm{H}), 3.90(\mathrm{~s}, 3 \mathrm{H}), 4.58(\mathrm{sep}, J=6.1 \mathrm{~Hz}, 1 \mathrm{H}), 4.64(\mathrm{t}, J=6.9 \mathrm{~Hz}, 2 \mathrm{H}), 6.50(\mathrm{~d}$, $J=4.2 \mathrm{~Hz}, 1 \mathrm{H}), 6.77(\mathrm{~s}, 1 \mathrm{H}), 6.97(\mathrm{~d}, J=4.2 \mathrm{~Hz}, 1 \mathrm{H}), 7.07(\mathrm{~s}, 1 \mathrm{H}), 9.50(\mathrm{~s}, 1 \mathrm{H}) .{ }^{13} \mathrm{C} \mathrm{NMR}(100 \mathrm{MHz}$, $\left.\mathrm{CDCl}_{3}\right): \delta 22.1,28.2,42.4,56.3,71.6,104.9,108.3,115.1,120.3,125.3,125.7,131.1,139.0,148.0,149.6$, 179.0. HRMS $(m / z)$ Calcd for $\mathrm{C}_{17} \mathrm{H}_{20} \mathrm{NO}_{3}\left[(\mathrm{M}+\mathrm{H})^{+}\right]$: 286.1443. Found: 286.1454. 
1-Bromo-5,6-dihydro-8-isopropoxy-9-methoxypyrrolo[2,1-a]isoquinoline-3-carbaldehyde

Under an argon atmosphere, NBS (62.9 mg, $0.353 \mathrm{mmol})$ was added portionwise to a solution of 13 (100 $\mathrm{mg}, 0.350 \mathrm{mmol})$ in THF $(5 \mathrm{~mL})$ at $\mathrm{rt}$, and the mixture was refluxed for $3 \mathrm{~h}$. After cooling to rt, the mixture was evaporated under reduced pressure. The residue was purified by column chromatography over silica gel $60 \mathrm{~N}$ (hexane-EtOAc $=3: 1$ ) to give 14 as a colorless solid (123 $\mathrm{mg}$, 96\%). Recrystallization from $\mathrm{Et}_{2} \mathrm{O}-$ hexane gave colorless plates. Mp 90.5-91 ${ }^{\circ} \mathrm{C}$. IR (KBr): 1660,1481 , 1399, 1208, 1126, $808 \mathrm{~cm}^{-1}$. ${ }^{1} \mathrm{H}$ NMR $\left(400 \mathrm{MHz} \mathrm{CDCl}_{3}\right): \delta 1.41(\mathrm{~d}, J=6.1 \mathrm{~Hz}, 6 \mathrm{H}), 2.97(\mathrm{t}, J=6.7 \mathrm{~Hz}$, 2H), $3.92(\mathrm{~s}, 3 \mathrm{H}), 4.60(\mathrm{sep}, J=6.1 \mathrm{~Hz}, 1 \mathrm{H}), 4.63(\mathrm{t}, J=6.7 \mathrm{~Hz}, 2 \mathrm{H}), 6.78(\mathrm{~s}, 1 \mathrm{H}), 6.99(\mathrm{~s}, 1 \mathrm{H}), 8.01(\mathrm{~s}$, $1 \mathrm{H}), 9.46(\mathrm{~s}, 1 \mathrm{H}) .{ }^{13} \mathrm{C} \mathrm{NMR}\left(100 \mathrm{MHz}, \mathrm{CDCl}_{3}\right): \delta 22.1,28.8,42.7,56.2,71.5,93.8,109.3,114.7,119.3$, 126.8, 127.1, 129.7, 134.0, 147.8, 149.0, 178.7. HRMS $(m / z)$ Calcd for $\mathrm{C}_{17} \mathrm{H}_{19} \mathrm{BrNO}_{3}\left[(\mathrm{M}+\mathrm{H})^{+}\right]$: 364.0548. Found: 364.0530 .

\section{5,6-Dihydro-8-isopropoxy-1-(3-isopropoxy-4-methoxyphenyl)-9-methoxypyrrolo[2,1-a]isoquinoline-}

3-carbaldehyde (15). Under an argon atmosphere, a mixture of 14 (600 mg, $1.65 \mathrm{mmol}), \mathbf{6}^{8 \mathrm{k}}(520 \mathrm{mg}$, $2.48 \mathrm{mmol}), \mathrm{Pd}\left(\mathrm{PPh}_{3}\right)_{4}(191 \mathrm{mg}, 0.165 \mathrm{mmol}), \mathrm{Na}_{2} \mathrm{CO}_{3}(1.15 \mathrm{~g}, 10.9 \mathrm{mmol}), \mathrm{DME}(40 \mathrm{~mL})$, and degassed water $(5 \mathrm{~mL})$ was refluxed for $16 \mathrm{~h}$. After cooling to $\mathrm{rt}$, the mixture was evaporated and the products were extracted with $\mathrm{CH}_{2} \mathrm{Cl}_{2}$. The extract was washed with water and brine, dried over $\mathrm{Na}_{2} \mathrm{SO}_{4}$, and evaporated. The residue was purified by flash chromatography over silica gel $60 \mathrm{~N}$ (toluene-EtOAc $=$ 10:1) to give 15 as a colorless solid (704 mg, 95\%). Recrystallization from $\mathrm{CH}_{2} \mathrm{Cl}_{2}$-hexane gave

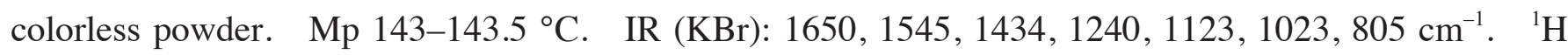
NMR (400 MHz, $\left.\mathrm{CDCl}_{3}\right): \delta 1.34(\mathrm{~d}, J=6.1 \mathrm{~Hz}, 6 \mathrm{H}), 1.38(\mathrm{~d}, J=6.1 \mathrm{~Hz}, 6 \mathrm{H}), 3.02(\mathrm{t}, J=6.7 \mathrm{~Hz}, 2 \mathrm{H})$, $3.40(\mathrm{~s}, 3 \mathrm{H}), 3.88(\mathrm{~s}, 3 \mathrm{H}), 4.49(\mathrm{sep}, J=6.1 \mathrm{~Hz}, 1 \mathrm{H}), 4.56(\mathrm{sep}, J=6.1 \mathrm{~Hz}, 1 \mathrm{H}), 4.65(\mathrm{t}, J=6.7 \mathrm{~Hz}, 2 \mathrm{H})$, $6.75(\mathrm{~s}, 1 \mathrm{H}), 6.88(\mathrm{~s}, 1 \mathrm{H}), 6.91(\mathrm{~s}, 1 \mathrm{H}), 6.92(\mathrm{~d}, J=7.9 \mathrm{~Hz}, 1 \mathrm{H}), 6.98(\mathrm{~s}, 1 \mathrm{H}), 6.99$ (dd, $J=1.9$ and $7.9 \mathrm{~Hz}$, 1H), $9.52(\mathrm{~s}, 1 \mathrm{H}) .{ }^{13} \mathrm{C} \mathrm{NMR}\left(100 \mathrm{MHz}, \mathrm{CDCl}_{3}\right): \delta 22.1,22.1,28.7,42.6,55.4,56.1,71.4,71.4,109.6$, $112.1,114.7,116.9,120.2$, 122.1, 123.2, 125.7, 126.7, 128.6, 129.6, 134.5, 147.3, 147.3, 148.7, 149.6, 179.1. $\operatorname{HRMS}(\mathrm{m} / \mathrm{z})$ Calcd for $\mathrm{C}_{27} \mathrm{H}_{32} \mathrm{NO}_{5}\left[(\mathrm{M}+\mathrm{H})^{+}\right]$: 450.2280 . Found: 450.2282.

\section{2-Bromo-5,6-dihydro-8-isopropoxy-1-(3-isopropoxy-4-methoxyphenyl)-9-methoxypyrrolo[2,1-a]iso-} quinoline-3-carbaldehyde (16). Under an argon atmosphere, a solution of NBS (29.7 mg, $0.167 \mathrm{mmol})$ in DMF (1.0 mL) was added dropwise to a solution of $15(50.0 \mathrm{mg}, 0.111 \mathrm{mmol})$ in DMF $(2.0 \mathrm{~mL})$ at $0{ }^{\circ} \mathrm{C}$. After stirring for $0.5 \mathrm{~h}$ at $0{ }^{\circ} \mathrm{C}$, the mixture was allowed to warm to rt. After stirring for $2 \mathrm{~h}$ at $\mathrm{rt}$, the mixture was quenched with $10 \%$ aqueous $\mathrm{Na}_{2} \mathrm{SO}_{3}$ and diluted with EtOAc. The product was washed with water and brine, dried over $\mathrm{Na}_{2} \mathrm{SO}_{4}$, and evaporated. The residue was purified by flash chromatography over silica gel $60 \mathrm{~N}$ (hexane-EtOAc $=3: 1$ ) to give $\mathbf{1 6}$ as a pale yellow solid $(44.5 \mathrm{mg}$, $76 \%$ ). Recrystallization from $\mathrm{CH}_{2} \mathrm{Cl}_{2}$-hexane gave a pale yellow powder. $\mathrm{Mp} 128-129{ }^{\circ} \mathrm{C}$. IR (KBr): 1643, 1465, 1370, 1240, 1135, 817, $728 \mathrm{~cm}^{-1} . \quad{ }^{1} \mathrm{H}$ NMR $\left(500 \mathrm{MHz}, \mathrm{CDCl}_{3}\right): \delta 1.36(\mathrm{~d}, J=6.1 \mathrm{~Hz}$, 
$6 \mathrm{H}), 1.36(\mathrm{~d}, J=6.1 \mathrm{~Hz}, 6 \mathrm{H}), 3.00(\mathrm{t}, J=6.8 \mathrm{~Hz}, 2 \mathrm{H}), 3.32(\mathrm{~s}, 3 \mathrm{H}), 3.89(\mathrm{~s}, 3 \mathrm{H}), 4.52(\mathrm{sep}, J=6.1 \mathrm{~Hz}$, 1H), 4.54 (sep, $J=6.1 \mathrm{~Hz}, 1 \mathrm{H}), 4.66$ (br s, 2H), 6.64 (s, 1H), 6.72 (s, 1H), 6.91 (dd, $J=1.9$ and $8.7 \mathrm{~Hz}$, $1 \mathrm{H}), 6.91(\mathrm{~d}, J=1.9 \mathrm{~Hz}, 1 \mathrm{H}), 6.96(\mathrm{~d}, J=8.7 \mathrm{~Hz}, 1 \mathrm{H}), 9.75(\mathrm{~s}, 1 \mathrm{H}) .{ }^{13} \mathrm{C} \mathrm{NMR}\left(125 \mathrm{MHz}, \mathrm{CDCl}_{3}\right): \delta$ 22.0, 22.1 , 28.3 42.4, 55.2, 56.1 , 71.3, 71.4, 109.6, 112.0, 114.5, 114.6, 118.1, 119.2, 122.6, 123.5, 125.0, 126.0, 126.5, 134.5, 147.4, 147.6, 148.7, 150.1, 179.4. HRMS $(m / z)$ Calcd for $\mathrm{C}_{27} \mathrm{H}_{31} \mathrm{BrNO}_{5}\left[(\mathrm{M}+\mathrm{H})^{+}\right]$: 528.1386. Found: 528.1399.

5,6-Dihydro-8-isopropoxy-1-(3-isopropoxy-4-methoxyphenyl)-2-[4-isopropoxy-5-methoxy-2-(methoxymethoxy)phenyl]-9-methoxypyrrolo[2,1-a]isoquinoline-3-carbaldehyde (4). According to the procedure described for the preparation of $\mathbf{1 5}, \mathbf{1 6}(341 \mathrm{mg}, 0.645 \mathrm{mmol}), \mathbf{7}^{8 \mathrm{k}}(262 \mathrm{mg}, 0.970 \mathrm{mmol})$, and $\mathrm{Pd}\left(\mathrm{PPh}_{3}\right)_{4}(74.9 \mathrm{mg}, 64.8 \mu \mathrm{mol})$ were reacted. After chromatographic purification over silica gel $60 \mathrm{~N}$ (hexane-EtOAc = 3:2), 4 was obtained as a pale yellow solid $(358 \mathrm{mg}, 82 \%)$. Recrystallization from $\mathrm{CH}_{2} \mathrm{Cl}_{2}$-hexane gave a yellow powder. $\mathrm{Mp} 103-105{ }^{\circ} \mathrm{C}$. IR $(\mathrm{KBr}): 1649,1429,1261,1213,1112$, $1027 \mathrm{~cm}^{-1} .{ }^{1} \mathrm{H}$ NMR $\left(500 \mathrm{MHz}, \mathrm{CDCl}_{3}\right): \delta 1.15(\mathrm{~d}, J=6.1 \mathrm{~Hz}, 3 \mathrm{H}), 1.18(\mathrm{~d}, J=6.1 \mathrm{~Hz}, 3 \mathrm{H}), 1.35(\mathrm{~d}, J=$ $6.1 \mathrm{~Hz}, 3 \mathrm{H}), 1.37(\mathrm{~d}, J=6.1 \mathrm{~Hz}, 3 \mathrm{H}), 1.38(\mathrm{~d}, J=6.1 \mathrm{~Hz}, 6 \mathrm{H}), 3.04(\mathrm{t}, J=6.6 \mathrm{~Hz}, 2 \mathrm{H}), 3.29$ (s, 3H), 3.34 $(\mathrm{s}, 3 \mathrm{H}), 3.61(\mathrm{~s}, 3 \mathrm{H}), 3.81(\mathrm{~s}, 3 \mathrm{H}), 4.25(\mathrm{sep}, J=6.1 \mathrm{~Hz}, 1 \mathrm{H}), 4.49(\mathrm{sep}, J=6.1 \mathrm{~Hz}, 1 \mathrm{H}), 4.55(\mathrm{sep}, J=$ $6.1 \mathrm{~Hz}, 1 \mathrm{H}), 4.65(\mathrm{br} \mathrm{s}, 1 \mathrm{H}), 4.73(\mathrm{~d}, J=6.6 \mathrm{~Hz}, 1 \mathrm{H}), 4.80$ (br s, 1H), $4.86(\mathrm{~d}, J=6.6 \mathrm{~Hz}, 1 \mathrm{H}), 6.56$ (s, $1 \mathrm{H}), 6.71(\mathrm{~d}, J=1.8 \mathrm{~Hz}, 1 \mathrm{H}), 6.76(\mathrm{~s}, 1 \mathrm{H}), 6.79(\mathrm{~d}, J=8.2 \mathrm{~Hz}, 1 \mathrm{H}), 6.79(\mathrm{~s}, 2 \mathrm{H}), 6.82(\mathrm{dd}, J=1.8$ and $8.2 \mathrm{~Hz}, 1 \mathrm{H}), 9.41(\mathrm{~s}, 1 \mathrm{H}) .{ }^{13} \mathrm{C} \mathrm{NMR}\left(125 \mathrm{MHz}, \mathrm{CDCl}_{3}\right): \delta 21.8,21.9,22.0,22.1,22.1,28.7,42.3,55.3$, 55.8, 56.1, 56.4, 71.1, 71.4, 71.4, 96.3, 105.2, 109.8, 111.7, 114.6, 116.4, 118.1, 120.3, 122.1, 123.4, $126.4,126.7,127.6,133.6,135.0,145.0,147.0,147.1,147.7,148.5,149.2,149.7,180.8$. HRMS $(m / z)$ Calcd for $\mathrm{C}_{39} \mathrm{H}_{48} \mathrm{NO}_{9}\left[(\mathrm{M}+\mathrm{H})^{+}\right]: 674.3329$. Found: 674.3347.

\section{5,6-Dihydro-2-(2-hydroxy-4-isopropoxy-5-methoxyphenyl)-8-isopropoxy-1-(3-isopropoxy-4-metho-} xyphenyl)-9-methoxypyrrolo[2,1-a]isoquinoline-3-carbaldehyde (17). To a solution of 4 (50.0 mg, $74.2 \mu \mathrm{mol})$ in $\mathrm{MeOH}(2.0 \mathrm{~mL})$ was added concd $\mathrm{HCl}(0.2 \mathrm{~mL})$ and the mixture was refluxed for $2 \mathrm{~h}$. After cooling to $\mathrm{rt}$, the mixture was evaporated. The products were extracted with $\mathrm{CH}_{2} \mathrm{Cl}_{2}$ and the extract was washed with water and brine, dried over $\mathrm{Na}_{2} \mathrm{SO}_{4}$, and evaporated. The residue was purified by column chromatography over silica gel 60N (hexane-EtOAc $=2: 1$ ) to give 17 as brown semisolid (34.2 $\mathrm{mg}, 73 \%)$. This compound was rather unstable and was used for the next reaction without further purification. IR (KBr): 3433, 1647, 1429, 1261, 1207, $1109 \mathrm{~cm}^{-1} . \quad{ }^{1} \mathrm{H}$ NMR (400 MHz, $\left.\mathrm{CDCl}_{3}\right): \delta 1.15$ $(\mathrm{d}, J=6.1 \mathrm{~Hz}, 3 \mathrm{H}), 1.19(\mathrm{~d}, J=6.1 \mathrm{~Hz}, 3 \mathrm{H}), 1.35(\mathrm{~d}, J=6.1 \mathrm{~Hz}, 3 \mathrm{H}), 1.37$ (d, $J=6.1 \mathrm{~Hz}, 3 \mathrm{H}), 1.38(\mathrm{~d}, J$ $=6.1 \mathrm{~Hz}, 6 \mathrm{H}), 3.01-3.10(\mathrm{~m}, 2 \mathrm{H}), 3.35(\mathrm{~s}, 3 \mathrm{H}), 3.68(\mathrm{~s}, 3 \mathrm{H}), 3.82(\mathrm{~s}, 3 \mathrm{H}), 4.26(\mathrm{sep}, J=6.1 \mathrm{~Hz}, 1 \mathrm{H}), 4.47$ (sep, $J=6.1 \mathrm{~Hz}, 1 \mathrm{H}), 4.57$ (sep, $J=6.1 \mathrm{~Hz}, 1 \mathrm{H}), 4.58$ (br s, 1H), 4.89 (br s, 2H), 6.46 (s, 1H), 6.62 (s, $1 \mathrm{H}), 6.71(\mathrm{~d}, J=1.8 \mathrm{~Hz}, 1 \mathrm{H}), 6.77(\mathrm{~s}, 1 \mathrm{H}), 6.79(\mathrm{~s}, 1 \mathrm{H}), 6.81(\mathrm{~d}, J=8.2 \mathrm{~Hz}, 1 \mathrm{H}), 6.85(\mathrm{dd}, J=1.8$ and $8.2 \mathrm{~Hz}, 1 \mathrm{H}), 9.41(\mathrm{~s}, 1 \mathrm{H}) .{ }^{13} \mathrm{C} \mathrm{NMR}\left(100 \mathrm{MHz}, \mathrm{CDCl}_{3}\right): \delta 21.7,21.9,22.0,22.1,22.1,28.7,42.4,55.3$, 
56.0, 56.7, 71.1, 71.2, 71.4, 103.2, 109.5, 109.8, 111.9, 114.6, 116.1, 117.9, 119.7, 122.3, 123.1, 126.3, $126.8,126.9,132.9,134.4,143.8,147.3,147.6,148.0,148.6,148.7,149.7,180.1$. HRMS $(\mathrm{m} / z)$ Calcd for $\mathrm{C}_{37} \mathrm{H}_{44} \mathrm{NO}_{8}\left[(\mathrm{M}+\mathrm{H})^{+}\right]$: 630.3067 . Found: 630.3054 .

\section{8,9-Dihydro-3,11-diisopropoxy-14-(3-isopropoxy-4-methoxyphenyl)-2,12-dimethoxy-6H-[1]benzo-} pyrano[4', $\left.3^{\prime}: 4,5\right]$ pyrrolo[2,1-a]isoquinolin-6-one (lamellarin $\mathrm{L}$ triisopropyl ether) (3). Under an argon atmosphere, a mixture of $17(30.3 \mathrm{mg}, 2.72 \mu \mathrm{mol})$, bromobenzene (6.0 $\mu \mathrm{L}, 56 \mu \mathrm{mol}), \operatorname{Pd}(\mathrm{OAc})_{2}$ (1.1 mg, $4.9 \mu \mathrm{mol})$, triphenylphosphine (3.9 mg, $15 \mu \mathrm{mol}), \mathrm{K}_{2} \mathrm{CO}_{3}(7.3 \mathrm{mg}, 53 \mu \mathrm{mol})$, and DMF $(2.0 \mathrm{~mL})$ was heated in a sealed tube at $120^{\circ} \mathrm{C}$ for $13 \mathrm{~h}$. After cooling to $\mathrm{rt}$, the mixture was diluted with water and extracted with $\mathrm{CH}_{2} \mathrm{Cl}_{2}$. The extract was washed with water and brine, dried over $\mathrm{Na}_{2} \mathrm{SO}_{4}$, and evaporated. The residue was purified by column chromatography over silica gel $60 \mathrm{~N}$ (hexane-EtOAc $=$ 2:1) to give 3 as a colorless solid (15.7 mg, 52\%). Recrystallization from $\mathrm{CH}_{2} \mathrm{Cl}_{2}-\mathrm{Et}_{2} \mathrm{O}$ gave a colorless powder. $\quad \operatorname{Mp} 207-208^{\circ} \mathrm{C}$. [lit. $\left.{ }^{8 \mathrm{k}} \mathrm{Mp} 206.5-207.5^{\circ} \mathrm{C}\right]$. IR (KBr): 1712, 1511, 1418, 1270, 1212, 1164, 1111, 1039, $939 \mathrm{~cm}^{-1} . \quad{ }^{1} \mathrm{H} \mathrm{NMR}\left(500 \mathrm{MHz}, \mathrm{CDCl}_{3}\right): \delta 1.33(\mathrm{~d}, J=6.1 \mathrm{~Hz}, 3 \mathrm{H}), 1.34(\mathrm{~d}, J=6.1 \mathrm{~Hz}, 3 \mathrm{H})$, $1.37(\mathrm{~d}, J=6.1 \mathrm{~Hz}, 3 \mathrm{H}), 1.37(\mathrm{~d}, J=6.1 \mathrm{~Hz}, 3 \mathrm{H}), 1.39$ (d, $J=6.1 \mathrm{~Hz}, 6 \mathrm{H}), 3.10(\mathrm{t}, J=6.8 \mathrm{~Hz}, 2 \mathrm{H}), 3.34$ (s, 3H), $3.43(\mathrm{~s}, 3 \mathrm{H}), 3.92(\mathrm{~s}, 3 \mathrm{H}), 4.48-4.58(\mathrm{~m}, 3 \mathrm{H}), 4.73-4.86(\mathrm{~m}, 2 \mathrm{H}), 6.66(\mathrm{~s}, 1 \mathrm{H}), 6.73(\mathrm{~s}, 1 \mathrm{H}), 6.77$ $(\mathrm{s}, 1 \mathrm{H}), 6.92(\mathrm{~s}, 1 \mathrm{H}), 7.05(\mathrm{~d}, J=1.6 \mathrm{~Hz}, 1 \mathrm{H}), 7.07(\mathrm{~d}, J=8.1 \mathrm{~Hz}, 1 \mathrm{H}), 7.09(\mathrm{dd}, J=1.6 \mathrm{and} 8.1 \mathrm{~Hz}, 1 \mathrm{H})$. ${ }^{13} \mathrm{C}$ NMR $\left(125 \mathrm{MHz}, \mathrm{CDCl}_{3}\right): \delta 21.8,21.9,22.0,22.1,28.7,42.5,55.1,55.5,56.3,71.3,71.4,71.4,103.5$, $104.9,109.2,110.4,112.7,113.7,114.8,114.8,117.9,120.3,123.7,126.4,128.0,128.3,136.0,146.0$, 146.5, 147.0, 147.3, 148.1, 148.7, 150.1, 155.7. HRMS $(\mathrm{m} / \mathrm{z})$ Calcd for $\mathrm{C}_{37} \mathrm{H}_{42} \mathrm{NO}_{8}\left[(\mathrm{M}+\mathrm{H})^{+}\right]: 628.2910$. Found: 628.2938. These physical and spectroscopic data are in good agreement with those previously reported. ${ }^{8 \mathrm{k}}$

\section{ACKNOWLEDGEMENTS}

This work was financially supported by a Grant-in-Aid for Scientific Research (B) (JSPS KAKENHI Grant Number 26293028) from the Japan Society for the Promotion of Science (JSPS).

\section{REFERENCES AND NOTES}

1. For recent reviews, see: (a) P. Cironi, F. Albericio, and M. Álvarez, Prog. Heterocycl. Chem., 2004, 16, 1; (b) C. Bailly, Curr. Med. Chem. - Anti-Cancer Agents, 2004, 4, 363; (c) S. T. Handy and Y. Zhang, Org. Prep. Proced. Int., 2005, 8, 411; (d) H. Fan, J. Peng, M. T. Hamann, and J.-F. Hu, Chem. Rev., 2008, 108, 264; (e) D. Pla, F. Albrecio, and M. Álvarez, Anti-Cancer Agents in Med. Chem., 2008, 8, 746; (f) J. Kluza, P. Marchetti, and C. Bailly, 'Modern Alkaloids: Structure, Isolation, Synthesis and Biology,' ed. by E. Fattorusso and O. Taglialatela-Scafati, Wiley-VCH, Weinheim, 
2008, pp. 171-187; (g) D. Pla, F. Albericio, and M. Álvarez, Med. Chem. Commun., 2011, 2, 689; (h) A.-L. Fan, W.-H. Lin, and Y.-X. Jia, J. Chin. Pharm. Sci., 2011, 20, 425; (i) T. Fukuda, F. Ishibashi, and M. Iwao, Heterocycles, 2011, 83, 491; (j) D. Imbri, J. Tauber, and T. Opatz, Mar. Drugs, 2014, 12,6142 .

2. (a) R. J. Anderson, D. J. Faulkner, H. Cun-heng, G. D. Van Duyne, and J. Clardy, J. Am. Chem. Soc., 1985, 107, 5492; (b) N. Lindquist and W. Fenical, J. Org. Chem., 1988, 53, 4570; (c) A. R. Carroll, B. F. Bowden, and J. C. Coll, Aust. J. Chem., 1993, 46, 489; (d) S. Urban, M. S. Butler, and R. J. Capon, Aust. J. Chem., 1994, 47, 1919; (e) S. Urban, L. Hobbs, J. N. A. Hooper, and R. J. Capon, Aust. J. Chem., 1995, 48, 1491; (f) S. Urban and R. J. Capon, Aust. J. Chem., 1996, 49, 711; (g) M. V. R. Reddy, D. J. Faulkner, Y. Venkateswarlu, and M. R. Rao, Tetrahedron, 1997, 53, 3457; (h) C. L. Cantrell, A. Groweiss, K. R. Gustafson, and M. R. Boyd, Nat. Prod.Lett., 1999, 14, 39; (i) R. A. Davis, A. R. Carroll, G. K. Pierens, and R. J. Quinn, J. Nat. Prod., 1999, 62, 419; (j) M. V. R. Reddy, M. R. Rao, D. Rhodes, M. S. T. Hansen, K. Rubins, F. D. Bushman, Y. Venkateswarlu, and D. J. Faulkner, J.Med. Chem., 1999, 42, 1901; (k) J. Ham and H. Kang, Bull. Korean Chem. Soc., 2002, 23, 163; (1) P. Krishnaiah, V. L. N. Reddy, G. Venkataramana, K. Ravinder, M. Srinivasulu, T. V. Raju, K. Ravikumar, D. Chandrasekar, S. Ramakrishna, and Y. Venkateswarlu, J. Nat. Prod., 2004, 67, 1168; (m) S. M. Reddy, M. Srinivasulu, N. Satyanarayana, A. K. Kondapi, and Y. Venkateswarlu, Tetrahedron, 2005, 61, 9242; (n) F. Plisson, X.-C. Huang, H. Zhang, Z. Khalil, and R. J. Capon, Chem. Asian J., 2012, 7, 1616; (o) H. Zhang, M. M. Conte, X.-C. Huang, Z. Khalil, and R. J. Capon, Org. Biomol. Chem., 2012, 10, 2656.

3. (a) A. R. Quesada, M. D. G. Grávalos, and J. L. F. Puentes, Br. J. Cancer, 1996, 74, 677; (b) F. Ishibashi, S. Tanabe, T. Oda, and M. Iwao, J. Nat. Prod., 2002, 65, 500; (c) C. P. Ridley, M. V. R. Reddy, G. Rocha, F. D. Bushman, and D. J. Faulkner, Bioorg. Med. Chem., 2002, 10, 3285; (d) C. Tardy, M. Facmpré, W. Laine, B. Baldeyrou, D. García-Gravalos, A. Francesch, C. Mateo, A. Pastor, J. A. Jiménez, I. Manzanares, C. Cuevas, and C. Bailly, Bioorg. Med. Chem., 2004, 12, 1697; (e) D. Pla, A. Marchal, C. A. Olsen, A. Francesch, C. Cuevas, F. Albericio, and M. Alvarez, J. Med. Chem., 2006, 49, 3257; (f) M. Chittchang, P. Batsomboon, S. Ruchirawat, and P. Ploypradith, ChemMedChem, 2009, 4, 457.

4. H. Kamiyama, Y. Kubo, H. Sato, N. Yamamoto, T. Fukuda, F. Ishibashi, and M. Iwao, Bioorg. Med. Chem., 2011, 19, 7541.

5. (a) M. Facompré, C. Tardy, C. Bal-Mahieu, P. Colson, C. Perez, I. Manzanares, C. Cuevas, and C. Bailly, Cancer Res., 2003, 63, 7392; (b) E. Marco, W. Laine, C. Tardy, A. Lansiaux, M. Iwao, F. Ishibashi, C. Bailly, and F. Gago, J. Med. Chem., 2005, 48, 3796.

6. (a) J. Kluza, M.-A. Gallego, A. Loyens, J.-C. Beauvillain, J.-M. F. Sousa-Faro, C. Cuevas, P. 
Marchetti, and C. Bailly, Cancer Res., 2006, 66, 3177; (b) M.-A. Gallego, C. Ballot, J. Kluza, N. Hajji, A. Martoriati, L. Castéra, C. Cuevas, P. Formstecher, B. Joseph, G. Kroemer, C. Bailly and P. Marchetti, Oncogene, 2008, 27, 1981; (c) C. Ballot, J. Kluza, A. Martoriati, U. Nyman, P. Formstecher, B. Joseph, C. Bailly, and P. Marchetti, Mol. Cancer Ther., 2009, 8, 3307; (d) C. Ballot, J. Kluza, S. Lancel, A. Martoriati, S. M. Hassoun, L. Mortier, J.-C. Vienne, G. Briand, P. Formstecher, C. Bailly, R. Nevière, and P. Marchetti, Apoptosis, 2010, 15, 769.

7. D. Baunbæk, N. Trinkler, Y. Ferandin, O. Lozach, P. Ploypradith, S. Rucirawat, F. Ishibashi, M. Iwao, and L. Meijer, Mar. Drugs, 2008, 6, 514.

8. (a) A. Heim, A. Terpin, and W. Steglich, Angew. Chem., Int. Ed. Engl., 1997, 36, 155; (b) M. Banwell, B. Flynn, and D. Hockless, Chem. Commun., 1997, 2259; (c) F. Ishibashi, Y. Miyazaki, and M. Iwao, Tetrahedron, 1997, 53, 5951; (d) C. Peschko, C. Winklhofer, and W. Steglich, Chem. Eur. J., 2000, 6, 1147; (e) S. Ruchirawat and T. Mutarapat, Tetrahedron Lett., 2001, 42, 1205; (f) M. Díaz, E. Guitián, and L. Castedo, Synlett, 2001, 1164; (g) P. Ploypradith, C. Mahidol, P. Sahakitpichan, S. Wongbundit, and S. Ruchirawat, Angew. Chem. Int. Ed., 2004, 43, 866; (h) S. T. Handy, Y. Zhang, and H. Bregman, J. Org. Chem., 2004, 69, 2362; (i) C. A. Olsen, N. Parera, F. Albericio, and M. Álvarez, Tetrahedron Lett., 2005, 46, 2041; (j) D. Pla, A. Marchal, C. A. Olsen, F. Albericio, and M. Álvarez, J. Org. Chem., 2005, 70, 8231; (k) N. Fujikawa, T. Ohta, T. Yamaguchi, T. Fukuda, F. Ishibashi, and M. Iwao, Tetrahedron, 2006, 62, 594; (1) P. Ploypradith, T. Petchmanee, P. Sahakitpichan, N. D. Litvinas, and S. Ruchirawat, J. Org. Chem., 2006, 71, 9440; (m) J. C. Liermann and T. Opatz, J. Org. Chem., 2008, 73, 4526; (n) L. Chen and M.-H. Xu, Adv. Synth. Catal., 2009, 351, 2005; (o) J. T. Gupton, B. C. Giglio, J. E. Eaton, E. A. Rieck, K. L. Smith, M. J. Keough, P. J. Barelli, L. T. Firich, J. E. Hempel, T. M. Smith, and R. P. F. Kanters, Tetrahedron, 2009, 65, 4283; (p) T. Ohta, T. Fukuda, F. Ishibashi, and M. Iwao, J. Org. Chem., 2009, 74, 8143; (q) K. Hasse, A. C. Willis, and M. G. Banwell, Eur. J. Org. Chem., 2011, 88; (r) Q. Li, J. Jiang, A. Fan, Y. Cui, and Y. Jia, Org. Lett., 2011, 13, 312; (s) B. L. Flynn and M. G. Banwell, Heterocycles, 2012, 84, 1141; (t) D. Imbri, J. Tauber, and T. Opatz, Chem. Eur. J., 2013, 19, 15080; (u) K. Ueda, K. Amaike, R. M. Maceiczyk, K. Itami, and J. Yamaguchi, J. Am. Chem. Soc., 2014, 136, 13226.

9. M. Komatsubara, T. Umeki, T. Fukuda, and M. Iwao, J. Org. Chem., 2014, 79, 529.

10. C. D'Silva and D. A. Walker, J. Org. Chem., 1998, 63, 6715.

11. C. Kashima, S. Hibi, T. Maruyama, and Y. Omote, Tetrahedron Lett., 1986, 27, 2131.

12. Vilsmeier-Haack reaction of $N$-substituted 2-arylpyrrole afforded the corresponding 5-formylated products, see: (a) M. A. Collins, V. Hudak, R. Bender, A. Fensome, P. Zhang, L. Miller, R. C. Winneker, Z. Zhang, Y. Zhu, J. Cohen, R. J. Unwalla, and J. Wrobel, Bioorg. Med. Chem. Lett., 2004, 14, 2185; (b) A. I. Mikhaleva, A. B. Zaitsev, A. V. Ivanov, E. Y. Schmidt, A. M. Vasil'tsov, 
and B. A. Trofimova, Tetrahedron Lett., 2006, 47, 3693; (c) M. Zajac, P. Hrobáric, P. Magdolen, P. Foltínova, and P. Zahradník, Tetrahedron, 2008, 64, 10605; (d) A. Deally, J. Claffey, B. Gleeson, M. Hogan, H. Müller-Bunz, S. Patil, D. F. O’Shea, and M. Tacke, Polyhedron, 2010, 29, 2445.

13. (a) G. A. Kraus and M. J. Taschner, J.Org. Chem., 1980, 45, 1175; (b) B. S. Bal, W. E. Childers Jr., and H. W. Pinnick, Tetrahedron, 1981, 37, 2091.

14. Y. Tamaru, Y. Yamada, K. Inoue, Y. Yamamoto, and Z. Yoshida, J. Org. Chem., 1983, 48, 1286.

15. In case $x \mathrm{~mol}$ of the amine hydrobromide $(\mathbf{9} \cdot \mathbf{H B r}$ ) was reacted to give $y \mathrm{~g}$ of a mixture of the indole (11) and the carbazole (12) and the molar fractions of $\mathbf{1 1}$ and $\mathbf{1 2}$ in the mixture were determined to be $N_{11}$ and $N_{12}$, the yields of $\mathbf{1 1}$ and $\mathbf{1 2}$ were estimated by using the following equations:

The yield of $\mathbf{1 1}\left(Y_{l 1}\right)=\frac{y N_{l l}}{x\left(M W_{l l} N_{l l}+M W_{l 2} N_{l 2}\right)} \times 100(\%)$

The yield of $12\left(Y_{12}\right)=\frac{y N_{12}}{x\left(M W_{11} N_{11}+M W_{12} N_{12}\right)} \times 100(\%)$

where $M W_{11}$ and $M W_{12}$ are molecular weights of $\mathbf{1 1}$ and $\mathbf{1 2 .}$ 